Vietnam Journal of Mechanics, VAST, Vol.39, No. 2 (2017), pp. $121-133$

DOI:10.15625/0866-7136/8444

\title{
FAST FOURIER TRANSFORMATION METHOD FOR COMPUTING THE PERMEABILITY OF PERIODIC POROUS MEDIA
}

\author{
Nguyen Trung Kien ${ }^{1, *}$, Nguyen Duy Hung ${ }^{2}$ \\ ${ }^{1}$ University of Transport and Communications, Hanoi, Vietnam \\ ${ }^{2}$ University of Transport and Communications-Campus in Ho Chi Minh City, Vietnam \\ *E-mail: ntkien@utc.edu.vn \\ Received June 23, 2016
}

\begin{abstract}
A Fourier-based method is adopted to determine the permeability of periodic porous media made up of a rigid skeleton saturated by viscous fluid. The flow, induced by a prescribed macroscopic gradient of pressure, adopts the Stokes equations with incorporating a condition of adherence at the surface of the solid. The permeability is determined by solving a linear problem on a unit cell for which we determine the local velocity fields due to a prescribed gradient of pressure. The method uses the Fourier Transformation and exact expressions of the periodic Green tensor in the Fourier space. It is shown that the resolution of the problem requires an introducing of undetermined forces acting within the solid phase.
\end{abstract}

Keywords: Porous media, permeability, homogenization, Fast Fourier Transform, viscous fluid.

\section{INTRODUCTION}

The determination of the permeability of porous media is of a fundamental importance in several practical problems in mechanics and civil engineering (biomechanic, petroleum, the flow in micro and nano systems, etc.). Different numerical approaches have been proposed in the literature for computing the permeability of periodic porous media. For instance, [1-4] use expansions along eigenfunctions. However, those studies reduce their analysis to some simple geometrical configurations such as the flow through regular arrays of cylinders or spheres. Standard numerical methods, such as the Finite Element one, can be used for computing the permeability of porous media, see for instance [5-9].

An alternative method to the Finite Element one has been proposed in the middle of the nineties by Moulinec et al. [10] in the context of linear elastic composites. This method uses an iterative scheme for computing the solution of the Lippmann-Schwinger

(C) 2017 Vietnam Academy of Science and Technology 
equation. The recurrence relation uses the exact expression of the Green tensor in the Fourier space and a representation of the elastic law in the real space. In this approach the elastic law is computed at each points of a regular grid, to avoid the difficulty due to meshing a multiphase unit cell. Particularly the method is adapted for complex microstructures, such as pixelized images, for which no additional treatment is required.

The Stokes equation present similarities with the equation of elasticity which justifies the use of the FFT based approach for computing the permeability. More precisely, the problem of the flow through a rigid skeleton can be interpreted in term of a linear viscous composite containing rigid inclusions. As classically in the framework of the homogenization of the periodic microstructures applied to porous media, the flow is generated by a prescribed macroscopic gradient of pressure which is interpreted as body forces for the equivalent linear viscous composite. However the application of the method using the FFT encounters a difficulty that does not appears in the context of linear composites. Indeed, the approach use a regular grid (such as in the Finite Difference Method) which requires to proceed to a continuation by continuity of the mechanical fields within the rigid inclusion. Within the solids there exists forces and couples which equilibrate the gradient of pressure and which can be only determined for simple geometrical configurations (such as the flow through regular array of cylinders or spheres).

\section{THE STOKES FLOW AND ITS EQUIVALENT COMPOSITE PROBLEM}

We consider a periodic porous medium saturated by a homogeneous Newtonian viscous fluid with the dynamic viscosity $\mu_{f}$. The periodic medium can be represented by a parallepipedic (rectangular for $2 \mathrm{D}$ problems) unit cell and three vectors (2 vectors for $2 \mathrm{D}$ problems) of spatial invariance. We define by $V_{f}$ the volume of the fluid, by $V_{s}$ the volume of the rigid skeleton and by $V=V_{f} \cup V_{s}$ the total volume of the unit cell. The surface of contact between the fluid and the solid is denoted $\partial V_{s}$. The flow is assumed to be incompressible and obey to the Stokes equations

$$
\begin{cases}\mu_{f} \Delta \underline{v}(\underline{x})+\nabla p(\underline{x})=0, & \forall \underline{x} \in V_{f} \\ \operatorname{div}(\underline{v}(\underline{x}))=0, & \forall \underline{x} \in V_{f} \\ \underline{v}(\underline{x})=0, & \forall \underline{x} \in \partial V_{s}\end{cases}
$$

In the above relations, $\underline{v}(\underline{x})$ and $p(\underline{x})$ are respectively the local velocity field and pressure. The flow of the fluid is generated by a prescribed macroscopic pressure gradient, denoted by $\underline{G}$. Consequently, the local pressure is decomposed as follows $p(\underline{x})=p^{*}(\underline{x})+\underline{G} . \underline{x}$ in which $p^{*}(\underline{x})$ is periodic. At the local scale, the components of the velocity field $\underline{v}(\underline{x})$ linearly depends on the components of the macroscopic pressure gradient

$$
\underline{v}(\underline{x})=A(\underline{x}) \cdot \underline{G}
$$

where $A_{i j}(x)$ depends on the position vector, $x$. As shown in the framework of the homogenization applied to porous media [11], the average value over the unit cell of the 
velocity field reads

$$
\underline{V}=<\underline{v}(\underline{x})>_{V}=-\frac{1}{\mu_{f}} K \cdot \underline{G},
$$

where $\underline{V}$ is called as macroscopic velocity field and $K$ is the permeability tensor.

$$
K=<A(\underline{x})>_{V} .
$$

The problem defined by Eqs. (1) presents a similarity with a problem of a linear viscous periodic composite with rigid inclusions

$$
\begin{cases}\dot{\varepsilon}(\underline{x})=\frac{1}{2}\left(\nabla \underline{v}(\underline{x})+\nabla^{t} \underline{v}(\underline{x})\right), & \forall \underline{x} \in V \\ \dot{\varepsilon}(\underline{x})=S(\underline{x}): \sigma(\underline{x}), & \forall \underline{x} \in V \\ \operatorname{div}(\sigma(\underline{x}))+\underline{f}(\underline{x})=0, & \forall \underline{x} \in V \\ \sigma(\underline{x}) \cdot \underline{n}, & \text { antiperiodic } \\ \underline{v}(\underline{x}), & \text { periodic }\end{cases}
$$

In the above equations, the local strain rate $\dot{\varepsilon}(\underline{x})$ and the local stress $\sigma(\underline{x})$ are periodic and their volumic mean over the unit cell is null: $\left\langle\dot{\varepsilon}(\underline{x})>_{V}=0\right.$ and $\langle\sigma(\underline{x})\rangle_{V}=0$. A continuation by continuity of the mechanical fields is effected within the solid volume, $V_{s}$. Classically, when the Finite Element Method (FEM) is used for solving the system of Eqs. (1), only the fluid phase is meshed. The surface $\partial V_{s}$ is considered as the domain boundary on which the Dirichlet condition, $\underline{v}=0$, is applied. The FFT method uses the discrete Fourier transform and then consist to discretize the unit cell by means of a regular grid as done with the Finite Difference Method. The points of that grid are located within the fluid or the solid phase which explain why a continuation by continuity of the strain and stress is needed.

The fourth order tensor $S(\underline{x})$ is given by

$$
\mathrm{S}(\underline{x})=\frac{1}{2 \mu(\underline{x})} \mathbb{K} \text { with }: \mu(\underline{x})=\left\{\begin{array}{l}
\mu_{f} \quad \forall \underline{x} \in V_{f} \\
+\infty \forall \underline{x} \in V_{s}
\end{array}\right.
$$

where $\mathbb{K}=\mathbb{I}-\mathbb{J}$ with $\mathbb{J}=\frac{1}{3} \boldsymbol{I} \otimes \boldsymbol{I}$ and $\mathbb{I}$ is the fourth order identity tensor. Note that in (5) the local stress contains a spherical part: $p(\underline{x}) \boldsymbol{I}$. Since $\mathbb{K}: \boldsymbol{I}=0$, the incompressibility $(\operatorname{tr}(\dot{\varepsilon})=0)$ is recovered within the fluid. In the solid phase, the components of the compliance $S(\underline{x})$ are infinite, the strain rate is then null. Classically, in the context of the homogenization of elastic periodic composite, prescribed macroscopic strain or stress are prescribed over the unit cell. In the present case, the macroscopic loading parameter is the macroscopic gradient of pressure (Fig. 1) which appears in (5) as body force

$$
\underline{f}(\underline{x})=\left\{\begin{array}{l}
\underline{G} \forall \underline{x} \in V_{f} \\
? \forall \underline{x} \in V_{s}
\end{array}\right.
$$




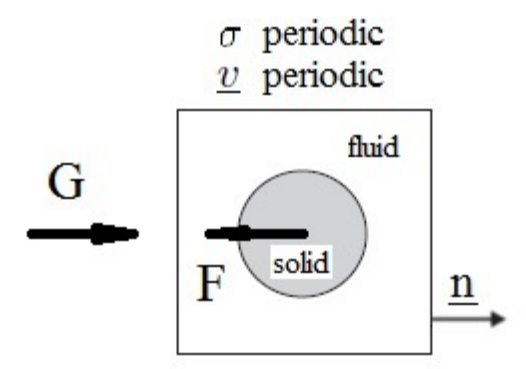

Fig. 1. Unit cell with boundary conditions: $G$ is the macroscopic gradient of pressure, $F$ is the body force in solid, $\underline{n}$ is the normal vector

In the above expression, the body force are undetermined within the solid phase (in $V_{s}$ ). This is discussed in the next section.

\section{THE FOURIER BASED METHOD}

Due to the similarity of the Stokes equations with the problem of linear composites we propose a similar method for computing the velocity field of the periodic porous medium. The method is stress based, since the equivalent periodic composite is made up of rigid inclusions (the strain based approach [10] diverge in that case).

In the present case, body forces are prescribed over the unit cell instead of macroscopic strain and stress, then we generalize the Lippmann-Schwinger equation in that context and we propose a FFT based iterative scheme for computing the solution of that integral equation.

\subsection{The Lippmann-Schwinger equation of the Stokes flow}

In (5), the compatibility and the equilibrium are local equations in the Fourier space since they reduce to algebraic relations. On the other hand, the local behavior, $\dot{\varepsilon}(\underline{x})=$ $\mathbb{S}(\underline{x}): \sigma(\underline{x})$, is local in the real space but it becomes non local in the Fourier space. To circumvent this difficulty, it is classically introduced a reference medium of compliance $\mathrm{S}^{0}$, and the eigenstrain $\eta(\underline{x})$ by

$$
\eta(\underline{x})=\left(\mathrm{S}(\underline{x})-\mathrm{S}^{0}\right): \sigma(\underline{x}) \quad \text { or } \quad \eta(\underline{\xi})=\left(\mathrm{S}(\underline{\xi})-\mathrm{S}^{0}\right) * \sigma(\underline{\xi}) .
$$

In the Fourier space, the system of Eqs. (5) can then be expressed in the form of (see Appendix A)

$$
\forall \underline{\xi} \neq 0:\left\{\begin{array}{l}
\mathbb{P}(\underline{\xi}): \sigma(\underline{\xi})+\boldsymbol{b}(\underline{\xi})=0 \\
\mathbb{Q}(\underline{\xi}): \dot{\varepsilon}(\underline{\xi})=0 \\
\dot{\varepsilon}(\underline{\xi})=S^{0}: \sigma(\underline{\xi})+\eta(\underline{\xi})
\end{array}\right.
$$


Eliminating the strain rate $\dot{\varepsilon}(\underline{\xi})$ in the above equations, we obtain

$$
\forall \underline{\xi} \neq 0:\left\{\begin{array}{l}
\mathbb{P}(\underline{\xi}): \sigma(\underline{\xi})+\boldsymbol{b}(\underline{\xi})=0 \\
\mathbb{Q}(\underline{\xi}):\left[S^{0}: \sigma(\underline{\xi})+\eta(\underline{\xi})\right]=0
\end{array}\right.
$$

Where tensors $\mathbb{P}(\underline{\xi})$ and $\mathbb{Q}(\underline{\xi})$ are defined by

$$
\mathbb{Q}(\underline{\xi})=\mathbb{E}_{1}(\underline{\xi})+\mathbb{E}_{3}(\underline{\xi}), \quad \mathbb{P}(\underline{\xi})=\mathbb{E}_{2}(\underline{\xi})+\mathbb{E}_{4}(\underline{\xi}) .
$$

In which the $\mathbb{E}_{n}(\underline{\xi})$ for $n=1 \ldots 4$ are the first four tensors of the Walpole basis [12] given by

$$
\left\{\begin{array}{l}
\mathbb{E}_{1}(\underline{\xi})=\frac{1}{2} \boldsymbol{k}^{\perp} \otimes \boldsymbol{k}^{\perp}, \mathbb{E}_{2}(\underline{\xi})=\boldsymbol{k} \otimes \boldsymbol{k} \\
\mathbb{E}_{3}(\underline{\xi})=\boldsymbol{k}^{\perp} \underline{\bar{\otimes}} \boldsymbol{k}^{\perp}-\mathbb{E}_{1}, \mathbb{E}_{4}(\underline{\xi})=\boldsymbol{k}^{\perp} \underline{\bar{\otimes}} \boldsymbol{k}+\boldsymbol{k} \underline{\bar{\otimes}} \boldsymbol{k}^{\perp} \\
\mathbb{E}_{5}(\underline{\xi})=\boldsymbol{k} \otimes \boldsymbol{k}^{\perp}, \mathbb{E}_{6}(\underline{\xi})=\boldsymbol{k}^{\perp} \otimes \boldsymbol{k}
\end{array}\right.
$$

where $\boldsymbol{k}$ and $\boldsymbol{k}^{\perp}$ are given by

$$
\boldsymbol{k}=\frac{1}{|\underline{\xi}|^{2}} \underline{\xi} \otimes \underline{\xi}, \boldsymbol{k}^{\perp}=I-k .
$$

Tensors $\mathbb{P}(\underline{\xi})$ and $\mathbb{Q}(\underline{\xi})$ are the two projectors

$$
\forall \underline{\xi} \neq 0:\left\{\begin{array}{l}
\mathbb{P}(\underline{\xi}): \mathbb{P}(\underline{\xi})=\mathbb{P}(\underline{\xi}), \mathbb{Q}(\underline{\xi}): \mathbb{Q}(\underline{\xi})=\mathbb{Q}(\underline{\xi}) \\
\mathbb{P}(\underline{\xi}): \mathbb{Q}(\underline{\xi})=\mathbb{Q}(\underline{\xi}): \mathbb{P}(\underline{\xi})=0 \\
\mathbb{I}=\mathbb{P}(\underline{\xi})+\mathbb{Q}(\underline{\xi})
\end{array}\right.
$$

From (10), it is observed that $\mathbb{P}(\underline{\xi}): \boldsymbol{b}(\underline{\xi})=\boldsymbol{b}(\underline{\xi})$ and $Q(\underline{\xi}): \boldsymbol{b}(\underline{\xi})=0$.

The two order tensor $\boldsymbol{b}(\underline{\xi})$, depends on the body force

$$
\boldsymbol{b}(\underline{\xi})=\frac{i}{\|\xi\|^{4}}\left[\underline{\xi} \otimes \underline{\xi} \underline{f}(\underline{\xi}) \cdot \underline{\xi}-(\underline{f}(\underline{\xi}) \otimes \underline{\xi}+\underline{\xi} \otimes \underline{f}(\underline{\xi}))\|\underline{\xi}\|^{2}\right] .
$$

A combination of the two relations of (10) gives

$$
\forall \underline{\xi} \neq 0:\left[\mathbb{Q}(\underline{\xi}): \mathbb{S}^{0}: \mathbb{Q}(\underline{\xi})\right]: \sigma(\underline{\xi})=\mathbb{Q}(\underline{\xi}):\left[\mathbb{S}^{0}: \boldsymbol{b}(\underline{\xi})-\boldsymbol{\eta}(\underline{\xi})\right] .
$$

The linear operator $\mathbb{Q}(\underline{\xi}): \mathbb{S}^{0}: \mathbb{Q}(\underline{\xi})$ is not invertible since $\mathbb{Q}(\underline{\xi})$ is a projector. However, it can be inverted according to $\mathbb{Q}(\underline{\xi})$, and its inverse is $\Delta^{0}(\underline{\xi})$, the Green tensor for the strain which can be expressed, in the Walpole basis, by

$$
\Delta^{0}(\underline{\xi})=\frac{2 \mu_{0}\left(3 \lambda_{0}+2 \mu_{0}\right)}{\lambda_{0}+2 \mu_{0}} \mathbb{E}_{1}(\underline{\xi})+2 \mu_{0} \mathbb{E}_{3}(\underline{\xi}) .
$$


where $\mu_{0}$ and $\lambda_{0}$ are Lame moduli of the reference medium. The solution of (15) is

$$
\mathbb{Q}(\underline{\xi}): \sigma(\underline{\xi})=\Delta^{0}(\underline{\xi}):\left[S^{0}: \boldsymbol{b}(\underline{\xi})-\boldsymbol{\eta}(\underline{\xi})\right] .
$$

Moreover, the projection along $\mathbb{P}(\underline{\xi})$ of the stress field $\sigma(\underline{\xi})$ is known (see Eq. (10)). The total stress is then given by

$$
\sigma(\underline{\xi})=-\boldsymbol{b}(\underline{\xi})+\Delta^{0}(\underline{\xi}):\left[S^{0}: \boldsymbol{b}(\underline{\xi})-\boldsymbol{\eta}(\underline{\xi})\right] .
$$

Note that the Green tensor for the stress, $\Delta^{0}(\underline{\xi})$ is related to the Green tensor for the strain, $\Gamma^{0}(\underline{\xi})$, by $\Delta^{0}(\underline{\xi})=\mathbb{C}^{0}-\mathbb{C}^{0}: \Gamma^{0}(\underline{\xi}): \mathbb{C}^{0}$. The above expression can then be also put into the form

$$
\sigma(\underline{\xi})=-\mathbb{C}^{0}: \Gamma^{0}(\underline{\xi}): \boldsymbol{b}(\underline{\xi})-\Delta^{0}(\underline{\xi}): \eta(\underline{\xi}) \text {. }
$$

Note that the expression of the Green tensor for the strain, $\Gamma^{0}(\underline{\xi})$, is, in the Walpole basis

$$
\Gamma^{0}(\underline{\xi})=\frac{1}{\lambda_{0}+2 \mu_{0}} \mathbb{E}_{2}(\underline{\xi})+\frac{1}{2 \mu_{0}} \mathbb{E}_{4}(\underline{\xi}) .
$$

Replacing $\boldsymbol{\eta}(\underline{\xi})$ in (19) by its expression (8), we finally obtain the searched equation

$$
\sigma(\underline{\xi})=-\mathbb{C}^{0}: \Gamma^{0}(\underline{\xi}): \boldsymbol{b}(\underline{\xi})-\Delta^{0}(\underline{\xi}):\left[\left(\mathbb{S}(\underline{\xi})-\mathbb{S}^{0}\right) * \sigma(\underline{\xi})\right] .
$$

Eq. (21) is the Lippmann-Schwinger equation for the stress, in the presence of the body forces $\underline{f}(\underline{x})$.

\subsection{The FFT-based iterative scheme}

As in [10], and along the lines of a method which was first introduced for composite conductors by Brown [13] and later by Kroner [14], the solution of the integral equation (21) can be expanded along Neumann series. Each term of the series is obtained by the following recurrence relation

$$
\begin{cases}\sigma^{i+1}(\underline{\xi})=-\mathbb{C}^{0}: \Gamma^{0}(\underline{\xi}): \boldsymbol{b}(\underline{\xi})-\Delta^{0}(\underline{\xi}):\left[\left(\mathbb{S}(\underline{\xi})-S^{0}\right) * \sigma^{i}(\underline{\xi})\right], & \text { for } \underline{\xi} \neq 0 \\ \sigma^{i+1}(\underline{\xi})=0, & \text { for } \underline{\xi}=0\end{cases}
$$

which is initiated by

$$
\sigma^{i=1}(\underline{\xi})=-\mathbb{C}^{0}: \Gamma^{0}(\underline{\xi}): \boldsymbol{b}(\underline{\xi}) .
$$

A simplification of the iterative scheme (22) is possible. The following relation hold at each iteration $i$

$$
\Delta^{0}(\underline{\xi}): \mathbb{S}^{0}: \sigma^{i}(\underline{\xi})=\sigma^{i}(\underline{\xi})+\mathbb{C}^{0}: \Gamma^{0}(\underline{\xi}): \boldsymbol{b}(\underline{\xi})
$$


The iterative scheme $(22)$ reduce to

$$
\begin{cases}\sigma^{i+1}(\underline{\xi})=\sigma^{i}(\underline{\xi})-\Delta^{0}(\underline{\xi}):\left(\mathrm{S}(\underline{\xi}) * \sigma^{i}(\underline{\xi})\right), & \text { for } \underline{\xi} \neq 0 \\ \sigma^{i+1}(\underline{\xi})=0, & \text { for } \underline{\xi}=0\end{cases}
$$

It is interesting to observe that the body forces does not appears in the iterative scheme (25). Then the obtained recurrence relation is equivalent to the one used in the context of elastic composites with prescribed macroscopic stress $[15,16]$. However the proposed algorithm for porous media differs from the one used for composites by the first term of the recurrence relation which depends on the body forces $f(\underline{x})$.

The exact Fourier transform is then replaced by the discrete Fourier transform which is computed by the FFT algorithm. The discrete wave vectors, $\underline{\xi}_{n}$, are taken from $n=-N$ to $n=N$, and by $\underline{x}_{n}$ we denote the position of the nodes of the regular grid in the real space. The algorithm is summarized below

$$
\begin{aligned}
& \sigma^{i}\left(\underline{x}_{n}\right)=\mathcal{F}^{-1}\left(\sigma^{i}\left(\underline{\xi}_{n}\right)\right) \\
& \mathcal{\varepsilon}^{i}\left(\underline{x}_{n}\right)=\mathbb{S}\left(\underline{x}_{n}\right): \sigma^{i}\left(\underline{x}_{n}\right) \\
& \dot{\varepsilon}^{i}\left(\underline{\xi}_{n}\right)=\mathcal{F}\left(\dot{\varepsilon}^{i}\left(\underline{x}_{n}\right)\right) \\
& \text { convergence test } \\
& \sigma^{i+1}\left(\underline{\xi}_{n}\right)=\sigma^{i}\left(\underline{\xi}_{n}\right)-\Delta^{0}\left(\underline{\xi}_{n}\right): \varepsilon^{i}\left(\underline{\xi}_{n}\right)
\end{aligned}
$$

Note that the stress complies with the equilibrium at each step $i$ of the iterative scheme. However, the strain rate complies with the compatibility only at convergence of the iterative scheme. The following convergence test is then used

$$
\frac{\left\|Q\left(\underline{\xi}_{n}\right): \dot{\varepsilon}\left(\underline{\xi}_{n}\right)\right\|}{\left\|\dot{\varepsilon}\left(\underline{\xi}_{n}\right)\right\|}<\epsilon,
$$

where $\|\bullet\|$ denote the Frobenius norm. Value $\epsilon=10^{-3}$ is used in the present work.

At convergence of the iterative scheme, one can compute the velocity associated to the strain rate field $\varepsilon^{i}\left(\underline{\xi}_{n}\right)$. Using the incompressibility condition $\left(\underline{v}\left(\underline{\xi}_{n}\right) \cdot \underline{\xi}_{n}=0\right)$, one has

$$
\begin{cases}\underline{v}\left(\underline{\xi}_{n}\right)=-\frac{2 i}{\left\|\underline{\xi}_{n}\right\|^{2}} \dot{\varepsilon}\left(\underline{\xi}_{n}\right) \cdot \underline{\xi}_{n}, & \text { for } \underline{\xi}_{n} \neq 0 \\ \underline{v}\left(\underline{\xi}_{n}\right)=?, & \text { for } \underline{\xi}_{n}=0\end{cases}
$$

At convergence of the iterative scheme, the velocity field is defined by its Fourier coefficients for all values of $\underline{\xi}_{n}$ except for $\underline{\xi}_{n}=0$. It means that the velocity field is defined up to an added constant. The reason for such a result is that the condition $\underline{v}(\underline{x})=0$ within 
the solid phase has never not been taken into account. As for the body force, the methodology leading to the identification of the macroscopic velocity field is depicted in the next section.

\section{VALIDATION OF THE APPROACH IN THE CASE OF REGULAR ARRAYS OF CYLINDERS AND SPHERES}

\subsection{Flow through regular arrays of cylinders}

In this section, it is proposed to apply the method in the context of flows through regular arrays of cylinders and to compare our numerical results with other solutions provided in the literature. The porous medium is then defined by a network of cylinders of radius $a$, aligned in the direction $0 x_{3}$. Fig. 2(a) represents the case of a tetragonal arrangement of the cylinders while Fig. 2(b) shows the case of the hexagonal arrangement. In the case of a tetragonal array of cylinders, the unit cell is defined by a square of half width $b$ containing a circular inclusion of radius $a$. In the case of a hexagonal array, the unit cell is defined by a rectangle with the half width $b$ and $b \sqrt{3}$ along directions $O x_{1}$ and $O x_{2}$ respectively $(b$ then represent the half distance between the axis of neighboring cylinders).

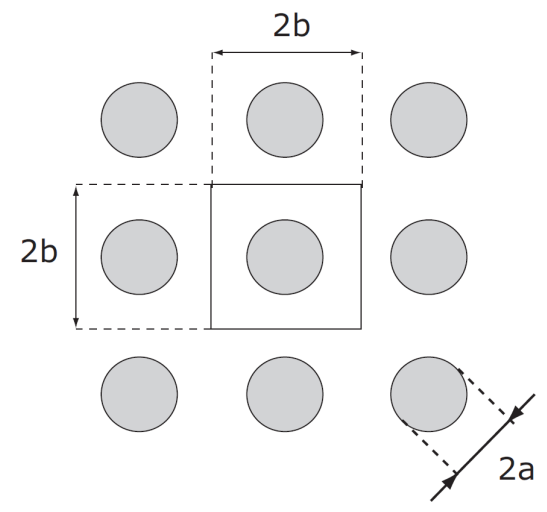

(a)

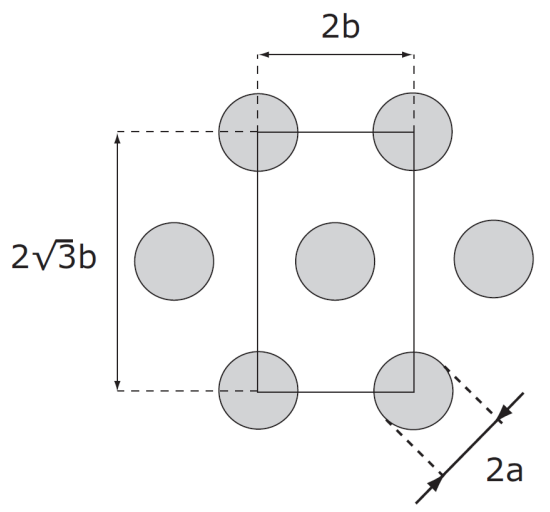

(b)

Fig. 2. Tetragonal (a) and Hexagonal (b) array of cylinders

For both configurations, the permeability tensor is defined by its nonzero components $K_{11}=K_{22}$ and $K_{33}$. In order to determine that components of the permeability, the two following loading cases are considered:

- A plane loading: $G_{1}=1, G_{2}=G_{3}=0$.

- An antiplane loading: $G_{1}=G_{2}=0, G_{3}=1$.

In the first loading case (the plane problem), the cylinders are kept fixed by applying, within the solid phase, the uniform body force $f_{1}=F_{1}$ and $f_{2}=f_{3}=0$. Indeed, the presence of forces along $O x_{2}$ or couples around $O x_{3}$ is excluded due to the symmetry of the unit cell. If we consider a uniform body force $f_{i}$ within the inclusion and considering 
the equilibrium of the unit cell, $<f_{i}>_{V}=0$, it is easy to check that $F_{1}$ is given by

$$
F_{1}=-\frac{\phi_{f}}{\phi_{s}} G_{1}
$$

where the volume fraction of the solid phase is $\phi_{s}=\pi R^{2} / S$ in the case of a tetragonal array, but is given by $\phi_{s}=2 \pi R^{2} / S$ in the case of a hexagonal array ( $S$ represents the area of the unit cell). It is recalled that $\phi_{f}=1-\phi_{s}$ is the volume fraction of the fluid. Similarly, in the second loading case (antiplane problem), the reaction of the cylinder is characterized by a force $F_{3}$, defined by

$$
F_{3}=-\frac{\phi_{f}}{\phi_{s}} G_{3}
$$

To summarize, the body force, in the real space reads

$$
\begin{aligned}
& \text { Plane problem: } f_{1}(\underline{x})=\left(1-\frac{1}{\phi_{s}} I_{s}(\underline{x})\right) G_{1}, f_{2}(\underline{x})=f_{3}(\underline{x})=0 . \\
& \text { Antiplane problem: } f_{1}(\underline{x})=f_{2}(\underline{x})=0, f_{3}(\underline{x})=\left(1-\frac{1}{\phi_{s}} I_{S}(\underline{x})\right) G_{3} .
\end{aligned}
$$

whereas in the Fourier space it reads

$$
\begin{aligned}
& \text { Plane problem: }\left\{\begin{array}{l}
\forall \underline{\xi} \neq 0: f_{1}(\underline{\xi})=-\frac{1}{\phi_{s}} G_{1} I_{s}(\underline{\xi}), \text { for } \underline{\xi}=0: f_{1}(\underline{\xi})=0 \\
f_{2}(\underline{\xi})=f_{3}(\underline{\xi})=0
\end{array}\right. \\
& \text { Antiplane problem: }\left\{\begin{array}{l}
f_{1}(\underline{\xi})=f_{2}(\underline{\xi})=0 \\
\forall \underline{\xi} \neq 0: f_{3}(\underline{\xi})=-\frac{1}{\phi_{s}} G_{3} I_{s}(\underline{\xi}), \text { for } \underline{\xi}=0: f_{3}(\underline{\xi})=0
\end{array}\right.
\end{aligned}
$$

The characteristic function of the solid phase, $I_{S}(\underline{\xi})$ can be exactly computed in the Fourier space since it is defined by simple geometrical volume (see Bonnet [16]).

At convergence of the iterative scheme (26), the velocity field is only known for $\underline{\xi}_{n} \neq 0$ (see (28)) but its volume average $\underline{V}$ is still unknown. The reason is that the condition $\underline{v}\left(\underline{x} \in V_{s}\right)=0$ has not been verified. Then we compute the periodic velocity field due the prescribed gradient of pressure $G_{1}$ and $G_{3}$ denoted $\underline{v}^{(1)}(\underline{x})$, $\underline{v}^{(3)}(\underline{x})$ and having a zero volume average. The total velocity field yields

$$
\underline{v}(\underline{x})=\underline{V}+\underline{v}^{(1)} G_{1}+\underline{v}^{(3)} G_{3} .
$$

The condition $\underline{v}\left(\underline{x} \in V_{s}\right)=0$, leads to the following expressions for the macroscopic components of the velocity

$$
\begin{aligned}
& V_{1}=-\underline{v}_{1}^{(1)}\left(\underline{x} \in V_{s}\right) G_{1}, \\
& V_{3}=-\underline{v}_{3}^{(3)}\left(\underline{x} \in V_{s}\right) G_{3},
\end{aligned}
$$

which finally leads to the identification of the components of the permeability tensor. 
On Fig. 3 we represent the normalized longitudinal and transverse permeability as function of the ratio $a / b$ for the tetragonal array of cylinder, $a$ being the radius of the cylinder and $b$ the half width of the unit cell (the cell being square in that example). On Fig. 3 the components of the permeability are normalized according to the area of the unit cell, $S=4 b^{2}$. As a validation purpose, the FFT results are compared with other existing results obtained from the literature by Wang [4], Sangani and Acrivos [1], Sparrow and Loeffler [17]).

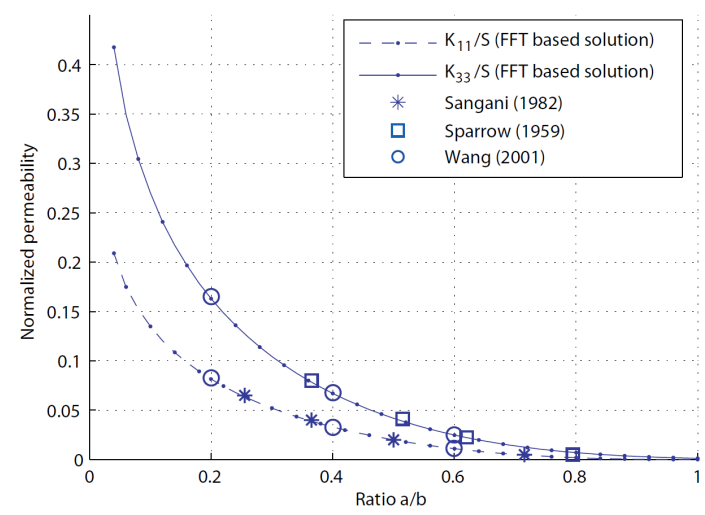

Fig. 3. Normalized permeabilities $K_{11} / S$ and $K_{33} / S$ as function of the ratio $a / b$ for the tetragonal array of cylinder of radius $a . b$ is the half distance between two neighboring cylinders

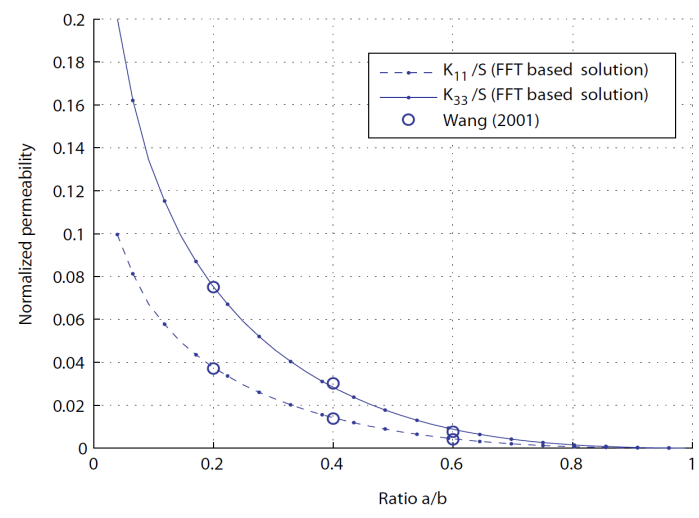

Fig. 4. Normalized permeabilities $K_{11} / S$ and $K_{33} / S$ as function of the ratio $a / b$ for the hexagonal array of cylinder of radius $a$. $b$ is the half distance between two neighboring cylinders

On Fig. 4 we represent the normalized longitudinal and transverse permeability as function of the ratio $a / b$ for the hexagonal array of cylinder, $a$ is the radius of the cylinders and $b$ is the half size of the unit cell in the direction $O x_{1}$. Along the direction $O x_{2}$, the half distance of the unit cell is $\sqrt{3} b$. The components of the permeability are still normalized according to the area of the unit cell, which is here: $S=4 \sqrt{3} b^{2}$. Again the FFT based numerical solutions are compared to the results provided by Wang [4].

\subsection{Flow through regular arrays of spheres}

It is now proposed to validate the approach by comparisons with existing results in the case of three-dimensional flows through arrays of spheres of radius $a$. The unit cell is defined by a cuboid of half width $b$. The following three classical configurations has been considered in our calculations:

- The simple cubic structure (SC). The unit cell is defined by a cube of side $2 b$ which contains a sphere of radius $a$ centered at $\underline{x}=0$. The volume fraction of the solid attained its maximum $\phi_{s}=\pi / 6$ when $a=b$.

- The centered cubic structure (CC). The basic cell contains two sphere of radius $a$. The maximum concentration of solid phase is $\sqrt{3} \pi / 8$ when $a=b$.

- The face-centered cubic structure (FCC). The unit contains 4 spheres of radius a and the concentration is $\sqrt{2} \pi / 6$. This is the most compact configuration. 
Due to the symmetries of the unit cell allows the permeability tensor is isotropic $K_{11}=K_{22}=K_{33}$ and $K_{i j}=0$ for $i \neq j$. The component $K_{11}$ is computed by applying the component $G_{1}$ of the gradient of pressure. As in the last example, the body force is fully described in the solid phase. In each inclusion the body force reads $f_{1}=F_{1}$ where $F_{1}$ is given by (29). The periodic, with a null volume average, is then computed for a prescribed value of $G_{1}$, denoted $\underline{v}_{1}^{(1)}\left(\underline{x} \in V_{s}\right)$. Following the method described in the case of cylinders, the macroscopic component of the macroscopic velocity field is computed as: $V_{1}=-\underline{v}_{1}^{(1)}\left(\underline{x} \in V_{s}\right) G_{1}$.

The case of a flow through networks of spheres has been studied by Sangani and Acrivos [2], Kadaksham et al. [18], Chapman and Higdon [19]. For reasons of clarity, our numerical solutions are compared only with the ones provided by Chapman and Higdon [19]. In Fig. 5 we represent the normalized permeability $K_{11} / 4 b^{2}$ as function of the ratio $a / b$ (where it is recalled that $a$ is the radius of the rigid spheres) and for each configurations (CS,CC and FCC).

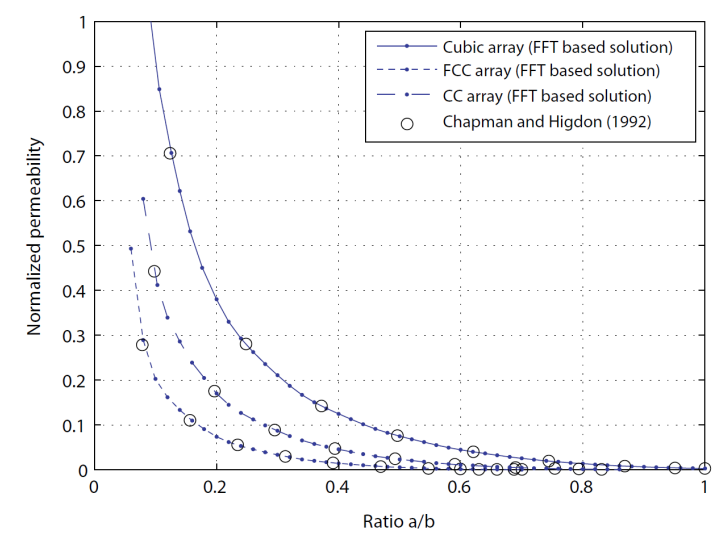

Fig. 5. Normalized permeability for the Simple Cubic (SC), Face Centered Cubic array (FCC) and centered cubic array (CC) of spheres of radius $a$

\section{CONCLUSION}

In the present paper we have proposed a numerical Fourier based method for computing the permeability of periodic porous media. The principle of the approach consist to re-write the problem of the Stokes flow into an equivalent problem of linear composite and to solve it by means of an iterative scheme similar to the ones introduced in $[10,16]$. It has been the resolution of the Stokes problem lead to some specifical difficulties which does not appears in the context of elastic composite. The flow through a porous media can be interpreted as an elastic problem with prescribed body forces which can be only determined for simple geometrical configurations. The implementation of the method and comparisons with results in the literature for plans and three-dimensional flow have illustrated the reliability of the approach. 


\section{ACKNOWLEDGMENT}

This research is supported by Vietnam National Foundation for Science and Technology Development (NAFOSTED) under grant number 107.02-2015.05

\section{REFERENCES}

[1] A. S. Sangani and A. Acrivos. Slow flow past periodic arrays of cylinders with application to heat transfer. International Journal of Multiphase Flow, 8, (3), (1982), pp. 193-206. doi:10.1016/0301-9322(82)90029-5.

[2] A. S. Sangani and A. Acrivos. Slow flow through a periodic array of spheres. International Journal of Multiphase Flow, 8, (4), (1982), pp. 343-360. doi:10.1016/0301-9322(82)90047-7.

[3] C. Y. Wang. Stokes flow through an array of rectangular fibers. International Journal of Multiphase Flow, 22, (1), (1996), pp. 185-194. doi:10.1016/0301-9322(95)00063-1.

[4] C. Y. Wang. Stokes flow through a rectangular array of circular cylinders. Fluid Dynamics Research, 29, (2), (2001), pp. 65-80. doi:10.1016/s0169-5983(01)00013-2.

[5] J. Barrere, J. P. Caltagirone, and O. Gipouloux. Détermination numérique de la perméabilité en milieu poreux périodique tridimensionnel. Comptes Rendus de l'Académie des Sciences. Série 2, Mécanique, Physique, Chimie, Sciences de l'univers, Sciences de la Terre, 310, (4), (1990), pp. 347352.

[6] D. Cioranescu, P. Donato, and H. I. Ene. Homogenization of the Stokes problem with nonhomogenous slip boundary conditions. Mathematical Methods in the Applied Sciences, 19, (11), (1996), pp. 857-881. doi:10.1002/(sici)1099-1476(19960725)19:11;857::aid-mma798_3.3.co;2-4.

[7] F. J. Alcocer, V. Kumar, and P. Singh. Permeability of periodic porous media. Physical Review E, 59, (1), (1999), p. 711. doi:10.1103/physreve.59.711.

[8] F. J. Alcocer and P. Singh. Permeability of periodic arrays of cylinders for viscoelastic flows. Physics of Fluids, 14, (7), (2002), pp. 2578-2581. doi:10.1063/1.1483301.

[9] E. Sawicki. Numerical investigations of the fluid flow through rotating porous media at both the microscopic scale and the macroscopic scale. PhD thesis, University of Joseph Fourier, Grenoble, (2004).

[10] H. Moulinec and P. Suquet. A fast numerical method for computing the linear and nonlinear mechanical properties of composites. Comptes Rendus de l'Académie des Sciences. Série II, Mécanique, Physique, Chimie, Astronomie, 318, (11), (1994), pp. 1417-1423.

[11] J. L. Auriault and E. Sanchez-Palencia. Study of macroscopic behavior of a deformable porous medium. Journal de Mcanique, 16, (4), (1997), pp. 575-603.

[12] L. Walpole. Elastic behavior of composite materials: theoretical foundations. Advances in Applied Mechanics, 21, (1981), pp. 169-242. doi:10.1016/s0065-2156(08)70332-6.

[13] W. F. Brown Jr. Solid mixture permittivities. The Journal of Chemical Physics, 23, (8), (1955), pp. 1514-1517. doi:10.1063/1.1742339.

[14] E. Kröner. Statistical continuum mechanics. Springer-Verlag, Wien, (1972).

[15] K. Bhattacharya and P. M. Suquet. A model problem concerning recoverable strains of shape-memory polycrystals. In Proceedings of the Royal Society of London A: Mathematical, Physical and Engineering Sciences, Vol. 461. The Royal Society, (2005), pp. 2797-2816. doi:10.1098/rspa.2005.1493.

[16] G. Bonnet. Effective properties of elastic periodic composite media with fibers. Journal of the Mechanics and Physics of Solids, 55, (5), (2007), pp. 881-899. doi:10.1016/j.jmps.2006.11.007.

[17] E. M. Sparrow and A. L. Loeffler. Longitudinal laminar flow between cylinders arranged in regular array. AIChE Journal, 5, (3), (1959), pp. 325-330. doi:10.1002/aic.690050315. 
[18] A. Kadaksham, S. B. Pillapakkam, and P. Singh. Permeability of periodic arrays of spheres. Mechanics Research Communications, 32, (6), (2005), pp. 659-665. doi:10.1016/j.mechrescom.2005.01.005.

[19] A. M. Chapman and J. J. L. Higdon. Oscillatory Stokes flow in periodic porous media. Physics of Fluids A: Fluid Dynamics, 4, (10), (1992), pp. 2099-2116. doi:10.1063/1.858507.

\section{Appendix A. Compatibility and equilibrium}

The relation yielding the strain rate tensor as function of the velocity field is, in the Fourier space

$$
\varepsilon(\underline{\xi})=i \underline{v}(\underline{\xi}) \stackrel{s}{\otimes} \underline{\xi},
$$

which can be also read $\forall \underline{\xi} \neq 0$

$$
\varepsilon(\underline{\xi}) \cdot k=\frac{i}{2}[\underline{v}(\underline{\xi}) \otimes \underline{\xi}+k(\underline{\xi} \cdot \underline{v}(\underline{\xi}))],
$$

where $k$ has been introduced in (12). It follows that

$$
\varepsilon(\underline{\xi}) \cdot \boldsymbol{k}+\boldsymbol{k} \cdot \varepsilon(\underline{\xi})=i \underline{v}(\underline{\xi}) \stackrel{s}{\otimes} \underline{\xi}+i \boldsymbol{k}(\underline{\xi} \cdot \underline{v}(\underline{\xi})) .
$$

Taking into account relation (35) and that $\operatorname{tr}(\varepsilon(\underline{\xi}))=i \underline{\xi} \cdot \underline{v}(\underline{\xi})$, the above expression can be put on the form

Or, equivalently

$$
\varepsilon(\underline{\xi}) \cdot k+k \cdot \varepsilon(\underline{\xi})=\varepsilon(\underline{\xi})+(\varepsilon(\underline{\xi}): k) k .
$$

$$
\boldsymbol{k}^{\perp} \cdot \varepsilon(\underline{\xi}) \cdot \boldsymbol{k}^{\perp}=0 .
$$

where $\boldsymbol{k}^{\perp}=\boldsymbol{I}-\boldsymbol{k}$. The above equation is exactly $\mathbb{Q}(\underline{\xi}): \varepsilon(\underline{\xi})=0$.

The equilibrium for $\sigma(\underline{\xi})$ reads, in the Fourier space

$$
i \sigma(\underline{\xi}) \cdot \underline{\xi}+\underline{f}(\underline{\xi})=0 .
$$

Which can be also put into the following equivalent form, $\forall \underline{\xi} \neq 0$

$$
\sigma(\underline{\xi}) \cdot k=\frac{i}{\|\underline{\xi}\|^{2}} f(\underline{\xi}) \otimes \underline{\xi} .
$$

It can then be easily shown that

$$
\mathbb{P}(\underline{\xi}): \sigma=\sigma(\underline{\xi}) \cdot k+k \cdot \sigma(\underline{\xi})-(\sigma(\underline{\xi}): k) k=-b(\underline{\xi}),
$$

where $\boldsymbol{b}(\underline{\xi})$ is defined by (14). 